\title{
The demand and supply of nutritional advice and guidance in Scottish family planning services
}

\author{
Sophie Tessier, BSc, MSc \\ Research Nutritionist, Department of Human Nutrition, University of Glasgow, Glasgow Royal Infirmary, Glasgow, UK
}

Susan E Morris, BAEcon, MSc, PhD, RPHNutr

Lecturer, Department of Applied Social Science, University of Stirling, Stirling, UK

Michael EJ Lean, MA MD, FRCP

Professor of Human Nutrition, Department of Human Nutrition, University of Glasgow, Glasgow Royal Infirmary, Glasgow, UK

(Accepted July 19 1999 )

\section{Summary}

Recent changes to the health services have led to an increased provision of clinical care in family planning clinics. While some women may only require contraceptive services, others may demand advice on a breadth of lifestyle issues, including diet and nutrition. Obesity affects $17 \%$ of women of childbearing age in Scotland and being overweight during pregnancy has significant health risks.

A postal survey of 227 nurses identified as working in family planning clinics in Scotland was conducted in 1998. After a mail shot and one reminder, a net response rate of $64 \%(n=145)$ was achieved. Overall, it was found that obesity was perceived as the most extensive problem in women of childbearing age. Seventy nurses (48\%) reported that, in addition to offering family planning services, they gave dietary advice 'frequently' or 'always' to their clients without being asked. There were differences in nutritionrelated activities in consultations between nurses offering family planning services only and those who routinely offered nutritional advice. Over half (61\%) of the nurses reported that they would give advice regarding weight management even if the patients were not seeking help, although there was no significant difference between the two comparison groups. In some cases, the nutritional advice offered to clients highlighted a deficit in training.

Most nutrition education came from diploma and/or training courses and scientific literature, followed by 'experience'. Those nurses already embracing a nutritional advice and guidance role were more interested in further nutrition training $(p=0.018)$ than the other nurses. However, overall, $67 \%$ of the nurses wished to train further in nutrition and weight management.

The findings suggest that family planning nurses should be supported to develop nutritional advice and guidance skills, and that there is a pressing need for training in public health nutrition and weight management for nurses working in family planning services.

\section{Keywords}

health professionals; health services; nutrition; postal surveys

\section{Key message point}

- There is a pressing need for specific training in public health nutrition and weight management to be made available for nurses working in family planning services.

\section{Introduction}

In women of child-bearing age, optimal nutritional status is crucial in the successful outcomes of pregnancy in successive generations. ${ }^{1}$ Public demand for nutrition information is increasing, and health professionals are perceived by the general public in the UK as reliable sources of dietary advice.

Since the NACNE report of $1983^{2}$ and the later COMA report in $1991,{ }^{3}$ advice about 'healthy' eating practices has been consistent for over 15 years. ${ }^{4}$ In pregnancy, nutritional requirements are increased and adequate dietary intakes should be encouraged by a well-balanced diet, across the whole range of nutrients. ${ }^{2}$ When nutrition is not optimal, risks of having premature, low birth-weight infants, or the occurrence of congenital malformations, are increased; folate deficiency being the most encountered attribute, especially if it occurs in the early weeks of the pregnancy. 5

Obesity, defined as body mass index (BMI) over $30 \mathrm{~kg} / \mathrm{m}^{2}$, affects $17.3 \%$ of women of child-bearing age. ${ }^{6}$ Increased risks of birth defects can occur among women who are underweight $\left(\mathrm{BMI}<18.5 \mathrm{~kg} / \mathrm{m}^{2}\right)^{7}$ and among those who are obese. ${ }^{8,9}$ Although the pattern of dietary intakes of these two groups is extremely different, both may be inadequate and can lead to severe nutrient deficiencies likely to impair pregnancy outcomes.

The primary health care sector has been recognised as an appropriate setting to educate the public towards healthier lifestyles and to implement dietary change. ${ }^{7}$ Family planning nurses (FPNs) are ideally placed to supply nutritional counselling (one to one dietary advice) to women of childbearing age. Women attending family planning clinics have been recognised as likely to have poor nutritional status as a result of poor lifestyle habits, such as negative attitude to health and poor diet. ${ }^{10}$ To fulfill their role as educators, health professionals must be able confidently to give appropriate nutrition information to their clients. However, the literature shows that the education of the primary health care (PHC) team in this area presents major gaps which can potentially mislead the public. ${ }^{11}$ Although the nutrition knowledge of the PHC team has been widely assessed, ${ }^{11-13}$ the knowledge of FPNs in this field remains relatively unexamined. In the literature, it appears that only one cross sectional survey in this important area has been conducted, in Sheffield. ${ }^{14}$ The study was limited by a small sample (16 health professionals based in three clinics) in one locality, and therefore is not expected to be representative of general family planning health professionals.

This paper reports on a survey of the nutrition in preconception care by FPNs across Scotland, and was conducted by the Department of Human Nutrition, University of Glasgow. The main aim of the study was to assess the perceived demand, and reported supply, of nutritional advice and guidance by family planning nurses in Scotland. Sources of nutritional education and reported interest in further training were also examined. 


\section{Method}

No listing of FPNs was accessible due to the occupational mobility of the profession, so a new database was generated from a call for information on the number of nurses in family planning clinics, and their names were obtained from every NHS trust in Scotland holding a family planning clinic.

A questionnaire collected demographic data (e.g. gender, age range, year of qualification in family planning and postcode of clinic). Sections covered current nutrition guidance practices during consultation, dietary knowledge with respect to obesity and pregnancy, the source of nutrition information and the need for further FPN training. Questions included: 'In consultation with your clients, how often do you give advice without being asked?' 'What dietary advice would you give to a woman contemplating pregnancy?' and 'What source of training or information has made the greatest contribution to your understanding of nutrition?'. The questionnaire was piloted with seven staff of a local hospital based family planning clinic, and changes were made where appropriate.

The first mail shot was sent on 09/03/97 $(n=227)$, and a reminder followed 4 weeks later. It was only possible to remind the nurses for whom a name was available $(n=55)$. Analysis was performed using SPSS for windows. ${ }^{15}$ Descriptive statistics, frequencies and Chi-square were carried out.

\section{Results}

One hundred and forty-five FPNs out of 227 participated in the study, giving a response rate of $64 \%$. The participants were divided into two comparison groups based on responses to the 'frequency of giving dietary advice without

Table 1 Socio-demographic characteristics of the sample $\dagger$

\begin{tabular}{|c|c|c|c|}
\hline & $\begin{array}{l}\text { Nurses offering } \\
\text { family planning } \\
\text { services and } \\
\text { nutritional guidance } \\
\qquad(\mathrm{n}=70)\end{array}$ & $\begin{array}{l}\text { Nurses offering } \\
\text { family planning } \\
\text { services only } \\
(n=69)\end{array}$ & $\begin{array}{c}\text { Total } \\
(\mathrm{n}=139)\end{array}$ \\
\hline \multicolumn{4}{|l|}{ Gender } \\
\hline Male & 0 & 1 & 1 \\
\hline Female & 69 & 67 & 136 \\
\hline Missing cases & 1 & 1 & 2 \\
\hline \multicolumn{4}{|l|}{ Age } \\
\hline Under 35 & 10 & 9 & 19 \\
\hline $35-54$ & 51 & 53 & 104 \\
\hline $55+$ & 8 & 6 & 14 \\
\hline Missing cases & 1 & 1 & 2 \\
\hline \multicolumn{4}{|l|}{ DEPCAT\# } \\
\hline 1 & 8 & 2 & 10 \\
\hline 2 & 5 & 6 & 11 \\
\hline 3 & 9 & 9 & 18 \\
\hline 4 & 21 & 20 & 41 \\
\hline 5 & 9 & 10 & 19 \\
\hline 6 & 16 & 18 & 34 \\
\hline 7 & - & 1 & 1 \\
\hline Missing cases & 2 & 3 & 5 \\
\hline \multicolumn{4}{|c|}{ Year of qualification } \\
\hline $1970-1979$ & 9 & 9 & 18 \\
\hline 1980-1989 & 29 & 25 & 54 \\
\hline 1990-1998 & 28 & 34 & 62 \\
\hline Missing cases & 4 & 1 & 5 \\
\hline
\end{tabular}

$\dagger 145$ nurses returned questionnaires and 139 gave a response to dietary advice questions.

\# Deprivation score ${ }^{21}$ (ranging from $1=$ most affluent to $7=$ most deprived). being asked' variable. FPNs who frequently or always gave advice were labelled as the 'nurses offering family planning services and nutritional advice and guidance,' and those who occasionally or never gave advice were labelled as 'nurses offering family planning services only'. Both groups had a mean year of qualification of 1988 (10 years prior to survey). There were no significant differences between the two comparison groups (see Table 1 for their socio-demographic characteristics).

The most extensive diet-related problem seen in consultation was obesity, being reported by $60 \%(n=87)$ of FPNs. Their reported nutrition guidance in these instances included foods in agreement with nutritional recommendations (i.e. decrease fat, sugar and salt; increase fresh fruit, vegetables and fibre). Six percent of FPNs gave misleading dietary advice for weight management. Three (2\%) FPNs reported that they would advise that carbohydrate and starchy foods such as potatoes and bread were fattening and should be restricted in the diet. One FPN recommended monitoring of protein intake, and one suggested increasing fatty foods.

In their nutritional advice and guidance with clients, the pro-active group was more likely to be asked about dietary advice by patients $(p=0.001)$, to weigh their clients using scales $(\mathrm{p}=0.27)$ and to promote exercise $(\mathrm{p}=0.008)$. There was no significant difference between the two groups with regards to the likelihood of giving advice on weight management if a client was not actively seeking help. Overall, 77.3\% ( $\mathrm{n}=106)$ claimed that they would advise clients on how to lose weight even if they were not seeking help.

An assessment of dietary advice regarding preconception and conception revealed four different types of advice. Food safety was of concern among $14.5 \%(n=21)$ of FPNs. Avoidance of pate, soft cheese, unpasteurised products, liver, vitamin A supplements, uncooked eggs and meat, and unwashed salad were commonly reported to be advised. Some foods were recommended to be increased in order to meet the optimal requirements of iron, calcium, vitamins and minerals during pregnancy.

Special attention was given to folic acid. A total of $74.5 \%$ $(n=108)$ of FPNs recommended folate supplementation at the dose of $400 \mathrm{mg} / \mathrm{day}$. Often a healthy/balanced diet was advocated, with plenty of fresh fruit and vegetables, cereals and wholemeal products. A few FPNs (3\%) recommended a regular meal pattern, including healthy snacks in between meals. Reported nutritional counselling included advising that alcohol and caffeine should be decreased, if not avoided. The last type of advice was relevant to lifestyle, and included exercising (e.g. swimming and walking) and stopping smoking.

The sources of greatest contribution to the nutritional guidance practices of FPNs are presented in Table 2. Literature (i.e. health promotion leaflets, governmental recommendation reports, research-based material) was the greatest source of nutritional information after the diploma, followed by the media.

The need for further training in nutrition was reported by $68 \%(\mathrm{n}=99)$ of FPNs; however, 'nurses offering family planning services and nutritional advice and guidance' were more likely to be interested in distance learning material $(p=0.018)$. Similarly, this group was also more likely to be interested in completing a training course on the role of nutrition in disease $(p=0.02)$, weight management $(p=0.03)$ and incorporating prevention into clinical practice $(\mathrm{p}=0.02)$. Neither group of nurses was interested in finding out more about the natural history and causes of obesity. 
Table 2 Sources of greatest contribution to nutritional education among FPNs $(n=145)$

\begin{tabular}{lrr}
\hline & $\mathrm{n}$ & $\%$ \\
\hline Diploma/training & 44 & 30.3 \\
Literature & 30 & 20.7 \\
Media (including women's magazines) & 17 & 11.7 \\
Courses & 15 & 10.3 \\
Weight wise course & 8 & 5.5 \\
Life & 8 & 5.5 \\
Work experience & 7 & 4.8 \\
Dietician & 7 & 4.8 \\
Personal attendance at slimming clubs & 6 & 4.1 \\
Missing cases & 3 & 2.0 \\
\hline
\end{tabular}

\section{Discussion}

This study aimed to produce data that would be of value to service managers by assessing the current demand by clients, and supply by nurses, of nutritional advice and guidance in Scottish family planning services. The problem of obesity was perceived to be extensive among family planning clients and although overall the dietary advice given by FPNs to adopt a healthier diet was appropriate, some confusion about appropriate dietary advice was evident.

The role of carbohydrates in the diet was not wholely clear to nurses, and there was a widespread misconception that carbohydrates contribute to weight gain and thus that their intake should be decreased. Likewise, only $16 \%$ of the general public think that it is important, and $44 \%$ fairly important, to increase intakes of starchy foods. ${ }^{16}$ Similar findings have been observed in a previous study conducted to assess dietetic knowledge among members of PHC teams in the Grampian region. ${ }^{11}$ Such nutritional guidance practices would be a cause for concern if their prevalence was high in the PHC team.

Nurses reported obesity as the major area to tackle and were likely to intervene even if clients were not seeking help. This suggests that the majority of FPNs are orientated towards 'preventative' services and are engaged in the promotion of healthy eating practices. It may be the case that patients were more likely to spontaneously seek dietary advice from those who are pro-active e.g. where posters and leaflets may be accessible. The literature indicates that educational material provided in waiting rooms has great potential for educating patients towards healthier lifestyles. ${ }^{17}$ The provision of information in the form of pamphlets, posters and videos in the waiting room encourages patients to ask informed questions. ${ }^{18}$

Guidance about nutrition in pregnancy was aimed at ensuring foetal growth rather than maternal weight management. However, efforts were made to emphasise the need for a healthy lifestyle. FPNs might take advantage of the pregnancy opportunity to deliver healthy messages in a stronger way, so that the future neonate can benefit from a good start in life. Again, the educational role of FPNs in ensuring adequate nutritional practices before and during pregnancy needs to be emphasised.

The present study confirmed the findings of a clinical audit of three clinics in Glasgow that 'clients now attend family planning clinics with a wide range of problems and the expectations that staff will be able to provide information, advice and counselling on these issues.' 19

Although FPNs appeared to be able to give general dietary advice, their knowledge about weight management was limited. This was strongly reflected by the high demand for distance learning materials from both FPN groups, especially on the topics of weight management and the incorporation of prevention into clinical practice.

\section{Conclusions}

The findings of this study suggest that FPNs should be helped to develop nutritional advice and guidance skills, and support the Scottish Diet Action Plan ${ }^{20}$ for continuing professional development to ensure that FPNs have the appropriate skills to do this.

\section{Statements on funding and competing interests}

Funding. None.

Competing interests. None.

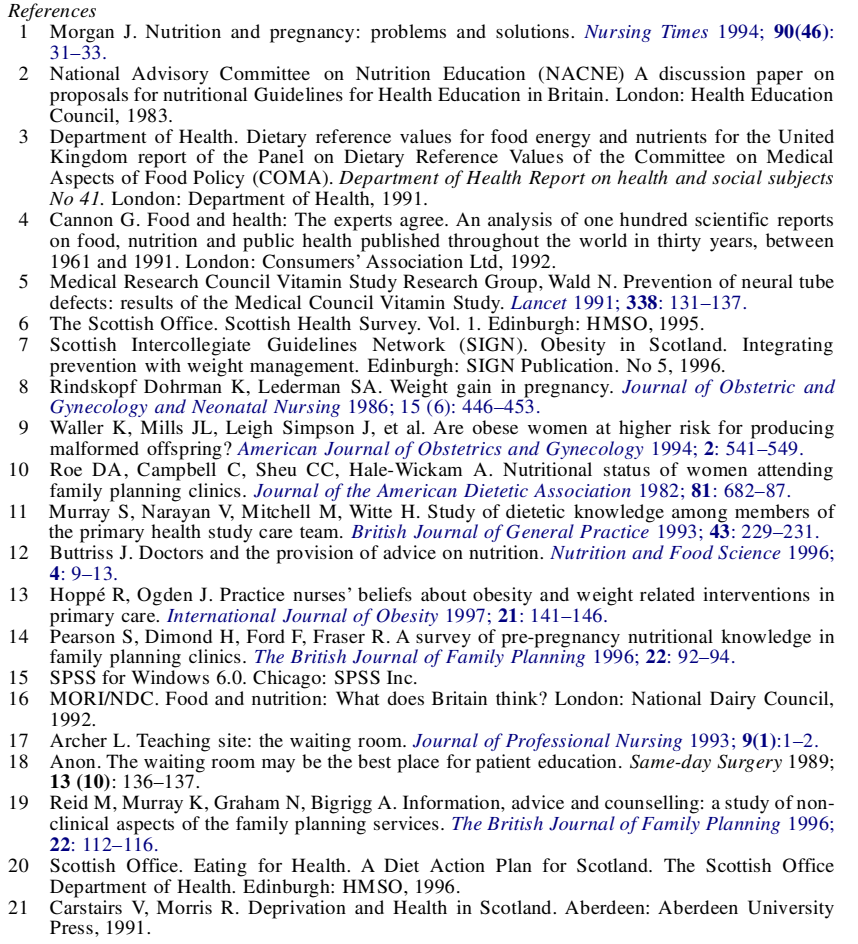

1 Morgan J. Nutrition and pregnancy: problems and solutions. Nursing Times 1994; 90(46): National Advisory Committee on Nutrition Education (NACNE) A discussion paper on Council, 1983.

Department of Health. Dietary reference values for food energy and nutrients for the Unite

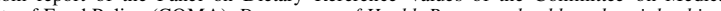
o 41. London: Department of Health, 1991. 961 and 1991. London: Consumers' Assocition Ltd, 199 . Medical Research Council Vitamin Study Research Group, Wald N. Prevention of neural tube efects: results of the Medical Council Vitamin Study. Lancet 1991; 338: 131-137.

Scottish In Ofice. Scottish Health Survey. Vol. (SIGN) Obesity in Scotland. Integratin

gain in pregnancy. Journal of Obstetric and

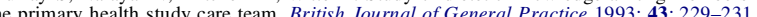
(2)

997: 21: 141-146.

itional knowledge in family planning clinics. The British Journal of Family Planning 1996; 22: 92-94.

Archer L. Teaching site: the waiting room. Journal of Professional Nursing 1993; 9(1):1-2.

Anon. The waiting room may be the best place for patient education. Same-day Surgery 198

clinical aspects of the family planning services. The British Journal of Family Planning 1996;

Carstairs V, Morris R. Deprivation and Health in Scotland. Aberdeen: Aberdeen University Press, 1991. 